\title{
Pacific
}

Journal of

Mathematics

\section{INTERLACING LOG-CONCAVITY \\ OF THE BOROS-MOLL POLYNOMIALS}

William Y. C. Chen, Larry X. W. Wang and Ernest X. W. Xia 


\title{
INTERLACING LOG-CONCAVITY OF THE BOROS-MOLL POLYNOMIALS
}

\author{
William Y. C. Chen, Larry X. W. Wang and Ernest X. W. Xia
}

We say a sequence $\left\{P_{m}(x)\right\}_{m \geq 0}$ of polynomials of degree $m$ with positive coefficients is interlacingly log-concave if the ratios of consecutive coefficients of $P_{m}(x)$ interlace the ratios of consecutive coefficients of $P_{m+1}(x)$ for any $m \geq 0$. Interlacing log-concavity of a sequence of polynomials is stronger than log-concavity of the polynomials themselves. We show that the BorosMoll polynomials are interlacingly log-concave. Furthermore, we give a sufficient condition for interlacing log-concavity which implies that some classical combinatorial polynomials are interlacingly log-concave.

\section{Introduction}

Let $\left\{P_{m}(x)\right\}_{m \geq 0}$ be a sequence of polynomials, where

$$
P_{m}(x)=\sum_{i=0}^{m} a_{i}(m) x^{m}
$$

is a polynomial of degree $m$. Let

$$
r_{i}(m)=\frac{a_{i}(m)}{a_{i+1}(m)} .
$$

We say that the sequence of polynomials $\left\{P_{m}(x)\right\}_{m \geq 0}$ is interlacingly log-concave if the ratios $r_{i}(m)$ interlace the ratios $r_{i}(m+1)$, that is,

$$
\begin{aligned}
r_{0}(m+1) \leq r_{0}(m) \leq r_{1}(m+1) & \leq r_{1}(m) \\
& \leq \cdots \leq r_{m-1}(m+1) \leq r_{m-1}(m) \leq r_{m}(m+1) .
\end{aligned}
$$

Recall that a sequence $\left\{a_{i}\right\}_{0 \leq i \leq m}$ of positive numbers is said to be log-concave if

$$
\frac{a_{0}}{a_{1}} \leq \frac{a_{1}}{a_{2}} \leq \cdots \leq \frac{a_{m-1}}{a_{m}} .
$$

It is obvious that interlacing log-concavity implies log-concavity.

This work was supported by the 973 Project, the PCSIRT Project, the Doctoral Program Fund of the Ministry of Education, and the National Science Foundation of China.

MSC2000: primary 05A20; secondary 33F10.

Keywords: interlacing log-concavity, log-concavity, Boros-Moll polynomial. 
The main objective of this paper is to prove the interlacing log-concavity of the Boros-Moll polynomials. For the background on these polynomials, see [Boros and Moll 1999a; 1999b; 1999c; 2001; 2004; Moll 2002; Amdeberhan and Moll 2009]. From now on, we use $P_{m}(x)$ to denote the Boros-Moll polynomial given by

$$
P_{m}(x)=\sum_{j, k}\left(\begin{array}{c}
2 m+1 \\
2 j
\end{array}\right)\left(\begin{array}{c}
m-j \\
k
\end{array}\right)\left(\begin{array}{c}
2 k+2 j \\
k+j
\end{array}\right) \frac{(x+1)^{j}(x-1)^{k}}{2^{3(k+j)}} .
$$

Boros and Moll [1999b] derived the following formula for the coefficient $d_{i}(m)$ of $x^{i}$ in $P_{m}(x)$ :

$$
d_{i}(m)=2^{-2 m} \sum_{k=i}^{m} 2^{k}\left(\begin{array}{c}
2 m-2 k \\
m-k
\end{array}\right)\left(\begin{array}{c}
m+k \\
k
\end{array}\right)\left(\begin{array}{l}
k \\
i
\end{array}\right) .
$$

In [Boros and Moll 1999c], they showed that the sequence $\left\{d_{i}(m)\right\}_{0 \leq i \leq m}$ is unimodal and that the maximum element appears in the middle. In other words,

$$
d_{0}(m)<d_{1}(m)<\cdots<d_{[m / 2]}(m)>d_{[m / 2]-1}(m)>\cdots>d_{m}(m) .
$$

They also established the unimodality by a different approach [Boros and Moll 1999a]; see also [Alvarez et al. 2001].

Moll [2002] conjectured that the sequence $\left\{d_{i}(m)\right\}_{0 \leq i \leq m}$ is log-concave. Kauers and Paule [2007] proved this conjecture based on recurrence relations found by using a computer algebra approach. Chen and Xia [2009] showed that the sequence $\left\{d_{i}(m)\right\}_{0 \leq i \leq m}$ satisfies the ratio monotone property which implies log-concavity and the spiral property. A combinatorial proof of the log-concavity of $P_{m}(x)$ was found by Chen, Pang and Qu [ $\geq 2011]$.

In addition to the Boros-Moll polynomials, we study polynomials whose coefficients satisfy triangular recurrence relations. It is easy to show that the binomial coefficients, the Narayana numbers and the Bessel numbers are interlacingly logconcave. We also give a sufficient condition for the interlacing log-concavity of a sequence of polynomials and prove that the rising factorials, the Bell polynomials and the Whitney polynomials are interlacingly log-concave.

\section{The interlacing log-concavity of $d_{i}(m)$}

In this section, we show that for $m \geq 2$, the Boros-Moll polynomials $P_{m}(x)$ are interlacingly log-concave.

Theorem 2.1. For $m \geq 2$ and $0 \leq i \leq m$, we have

$$
\begin{aligned}
d_{i}(m) d_{i+1}(m+1) & >d_{i+1}(m) d_{i}(m+1), \\
d_{i}(m) d_{i}(m+1) & >d_{i-1}(m) d_{i+1}(m+1) .
\end{aligned}
$$


The proof relies on recurrence relations derived in [Kauers and Paule 2007]:

(6) $\quad d_{i}(m+1)=\frac{m+i}{m+1} d_{i-1}(m)+\frac{(4 m+2 i+3)}{2(m+1)} d_{i}(m), \quad 0 \leq i \leq m+1$,

(7) $\quad d_{i}(m+1)=\frac{(4 m-2 i+3)(m+i+1)}{2(m+1)(m+1-i)} d_{i}(m)$

$$
-\frac{i(i+1)}{(m+1)(m+1-i)} d_{i+1}(m), \quad 0 \leq i \leq m,
$$

(8) $\quad d_{i}(m+2)=\frac{-4 i^{2}+8 m^{2}+24 m+19}{2(m+2-i)(m+2)} d_{i}(m+1)$

$$
-\frac{(m+i+1)(4 m+3)(4 m+5)}{4(m+2-i)(m+1)(m+2)} d_{i}(m), \quad 0 \leq i \leq m+1,
$$

and for $0 \leq i \leq m+1$,

(9) $(m+2-i)(m+i-1) d_{i-2}(m)-(i-1)(2 m+1) d_{i-1}(m)+i(i-1) d_{i}(m)=0$.

Moll [2007] independently derived the recurrence relations (6) and (9) from which the other two relations can be easily deduced.

To prove Theorem 2.1(4), we need the following lemma.

Lemma 2.2. Assume that $m \geq 2$. For $0 \leq i \leq m-2$, we have

$$
\frac{d_{i}(m)}{d_{i+1}(m)}<\frac{(4 m+2 i+3) d_{i+1}(m)}{(4 m+2 i+7) d_{i+2}(m)} .
$$

Proof. We proceed by induction on $m$. When $m=2$, it is easy to check that the result holds. Assume that the lemma is valid for $n$, namely,

$$
\frac{d_{i}(n)}{d_{i+1}(n)}<\frac{(4 n+2 i+3) d_{i+1}(n)}{(4 n+2 i+7) d_{i+2}(n)}, \quad 0 \leq i \leq n-2 .
$$

We aim to show that (10) holds for $n+1$, that is,

$$
\frac{d_{i}(n+1)}{d_{i+1}(n+1)}<\frac{(4 n+2 i+7) d_{i+1}(n+1)}{(4 n+2 i+11) d_{i+2}(n+1)}, \quad 0 \leq i \leq n-1 .
$$

From the recurrence relation (6), we deduce that, for $0 \leq i \leq n-1$,

$$
\begin{aligned}
&(2 i+4 n+7) d_{i+1}^{2}(n+1)-(2 i+4 n+11) d_{i}(n+1) d_{i+2}(n+1) \\
&=(2 i+4 n+7)\left(\frac{i+n+1}{n+1} d_{i}(n)+\frac{2 i+4 n+5}{2(n+1)} d_{i+1}(n)\right)^{2} \\
&-(2 i+4 n+11)\left(\frac{i+n+2}{n+1} d_{i+1}(n)+\frac{2 i+4 n+7}{2(n+1)} d_{i+2}(n)\right) \\
& \times\left(\frac{n+i}{n+1} d_{i-1}(n)+\frac{2 i+4 n+3}{2(n+1)} d_{i}(n)\right)
\end{aligned}
$$




$$
=\frac{A_{1}(n, i)+A_{2}(n, i)+A_{3}(n, i)}{4(n+1)^{2}},
$$

where $A_{1}(n, i), A_{2}(n, i)$ and $A_{3}(n, i)$ are given by

$$
\begin{gathered}
\begin{array}{c}
A_{1}(n, i)=4(2 i+4 n+7)(i+n+1)^{2} d_{i}^{2}(n) \\
-4(n+i)(2 i+4 n+11)(i+n+2) d_{i+1}(n) d_{i-1}(n), \\
A_{2}(n, i)=(2 i+4 n+7)(2 i+4 n+5)^{2} d_{i+1}^{2}(n) \\
-(2 i+4 n+3)(2 i+4 n+11)(2 i+4 n+7) d_{i}(m) d_{i+2}(n), \\
A_{3}(n, i)=\left(8 i^{3}+40 i^{2}+58 i+32 n^{3}+42 n+80 n^{2}+120 n i+40 i^{2} n+64 n^{2} i+8\right) \\
\cdot d_{i+1}(n) d_{i}(n)-2(n+i)(2 i+4 n+11)(2 i+4 n+7) d_{i+2}(n) d_{i-1}(n) .
\end{array}
\end{gathered}
$$

We will show that $A_{1}(n, i), A_{2}(n, i)$ and $A_{3}(n, i)$ are all positive for $0 \leq i \leq n-2$. By the induction hypothesis (11), we find that for $0 \leq i \leq n-2$,

$$
\begin{aligned}
A_{1}(n, i)> & 4(2 i+4 n+7)(i+n+1)^{2} d_{i}^{2}(n) \\
& -4(n+i)(2 i+4 n+11)(i+n+2) \frac{(4 n+2 i+1)}{(4 n+2 i+5)} d_{i}^{2}(n) \\
= & \frac{35+96 n+72 i+64 n i+40 n^{2}+28 i^{2}}{2 i+4 n+5} d_{i}^{2}(n), \\
A_{2}(n, i)>(2 i+4 n+7)(2 i+4 n+5)^{2} d_{i+1}^{2}(n) & \quad-(2 i+4 n+3)(2 i+4 n+11)(2 i+4 n+7) \frac{(4 n+2 i+3)}{(4 n+2 i+7)} d_{i+1}^{2}(n) \\
= & (40 i+80 n+76) d_{i+1}^{2}(n),
\end{aligned}
$$

which are both positive. Also by the induction hypothesis (11), we see that

$$
d_{i}(n) d_{i+1}(n)>\frac{(2 i+4 n+5)(2 i+4 n+7)}{(2 i+4 n+3)(2 i+4 n+1)} d_{i-1}(n) d_{i+2}(n),
$$

for $0 \leq i \leq n-2$. This implies that

$$
\begin{aligned}
& A_{3}(n, i) \\
& >\left(8 i^{3}+40 i^{2}+58 i+32 n^{3}+42 n+80 n^{2}+120 n i+40 i^{2} n+64 n^{2} i+8\right) d_{i+1}(n) d_{i}(n) \\
& \quad-2(n+i)(2 i+4 n+11)(2 i+4 n+7) \frac{(4 n+2 i+3)(4 n+2 i+1)}{(4 n+2 i+5)(4 n+2 i+7)} d_{i+1}(n) d_{i}(n) \\
& =8 \frac{5+22 n+30 i+44 n i+24 n^{2}+16 i^{2}}{2 i+4 n+5} d_{i+1}(n) d_{i}(n),
\end{aligned}
$$


which is positive for $0 \leq i \leq n-2$. Hence the inequality (12) holds for $0 \leq i \leq n-2$. It remains to show that (12) is true for $i=n-1$, that is,

$$
\frac{d_{n-1}(n+1)}{d_{n}(n+1)}<\frac{(6 n+5) d_{n}(n+1)}{(6 n+9) d_{n+1}(n+1)} .
$$

From (2) it follows that

$$
\begin{aligned}
d_{n}(n+1) & =2^{-n-2}(2 n+3)\left(\begin{array}{c}
2 n+2 \\
n+1
\end{array}\right), \\
d_{n+1}(n+1) & =\frac{1}{2^{n+1}}\left(\begin{array}{c}
2 n+2 \\
n+1
\end{array}\right), \\
d_{n}(n+2) & =\frac{(n+1)\left(4 n^{2}+18 n+21\right)}{2^{n+4}(2 n+3)}\left(\begin{array}{c}
2 n+4 \\
n+2
\end{array}\right) .
\end{aligned}
$$

Consequently,

$$
\frac{d_{n-1}(n+1)}{d_{n}(n+1)}=\frac{n\left(4 n^{2}+10 n+7\right)}{2(2 n+1)(2 n+3)}<\frac{(2 n+3)(6 n+5)}{2(6 n+9)}=\frac{(6 n+5) d_{n}(n+1)}{(6 n+9) d_{n+1}(n+1)} .
$$

This completes the proof.

We can now prove Theorem 2.1(4). In fact, we shall prove a stronger inequality.

Lemma 2.3. Assume that $m \geq 2$. For $0 \leq i \leq m-1$, we have

$$
\frac{d_{i}(m)}{d_{i+1}(m)}>\frac{(2 i+4 m+5) d_{i}(m+1)}{(2 i+4 m+3) d_{i+1}(m+1)} .
$$

Proof. By Lemma 2.2, we have for $0 \leq i \leq m-1$,

$$
d_{i}^{2}(m)>\frac{2 i+4 m+5}{2 i+4 m+1} d_{i-1}(m) d_{i+1}(m) .
$$

From (19) and the recurrence relation (6), for $0 \leq i \leq m-1$,

$$
\begin{aligned}
& d_{i+1}(m+1) d_{i}(m)-\frac{2 i+4 m+5}{2 i+4 m+3} d_{i+1}(m) d_{i}(m+1) \\
& =\frac{2 i+4 m+5}{2(m+1)} d_{i+1}(m) d_{i}(m)+\frac{i+m+1}{m+1} d_{i}(m)^{2} \\
& \quad-\frac{2 i+4 m+5}{2 i+4 m+3}\left(\frac{2 i+4 m+3}{2(m+1)} d_{i}(m) d_{i+1}(m)+\frac{i+m}{m+1} d_{i-1}(m) d_{i+1}(m)\right) \\
& =\frac{i+m+1}{m+1} d_{i}^{2}(m)-\frac{(4 m+2 i+5)(m+i)}{(4 m+2 i+3)(m+1)} d_{i-1}(m) d_{i+1}(m) \\
& > \\
& =\left(\frac{m+1+i}{m+1}-\frac{(4 m+2 i+1)(m+i)}{(4 m+2 i+3)(m+1)}\right) d_{i}^{2}(m) \\
& =\frac{6 m+4 i+3}{(4 m+2 i+3)(m+1)} d_{i}^{2}(m)>0,
\end{aligned}
$$

which yields (18). 
We now turn to the proof of Theorem 2.1(5).

Lemma 2.4. Assume that $m \geq 2$. For $0 \leq i \leq m-1$, we have

$$
\frac{d_{i}(m)}{d_{i+1}(m)}<\frac{d_{i+1}(m+1)}{d_{i+2}(m+1)}
$$

Proof. We proceed by induction on $m$. It is easy to check the lemma holds for $m=2$. Assume that the lemma is true for $n \geq 2$, that is,

$$
\frac{d_{i}(n)}{d_{i+1}(n)}<\frac{d_{i+1}(n+1)}{d_{i+2}(n+1)}, \quad 0 \leq i \leq n-1 .
$$

It will be shown that the theorem holds for $n+1$, that is,

$$
\frac{d_{i}(n+1)}{d_{i+1}(n+1)}<\frac{d_{i+1}(n+2)}{d_{i+2}(n+2)}, \quad 0 \leq i \leq n .
$$

Recall that the sequence $\left\{d_{i}(n+1)\right\}_{0 \leq i \leq n+1}$ is unimodal. Furthermore, from (3) or the ratio monotone property [Chen and Xia 2009], the maximum element appears in the middle, namely, $d_{i}(n+1)<d_{i+1}(n+1)$ when $0 \leq i \leq[(n+1) / 2]-1$ and $d_{i}(n+1)>d_{i+1}(n+1)$ when $[(n+1) / 2] \leq i \leq n$.

Showing (22) for $0 \leq i \leq n-1$ breaks into two cases.

The first case is $d_{i}(n+1)<d_{i+1}(n+1)$, namely, $0 \leq i \leq[(n+1) / 2]-1$. From the recurrence relation (6), we find that for $0 \leq i \leq[(n+1) / 2]-1$,

$$
\begin{aligned}
& d_{i+1}(n+1) d_{i+1}(n+2)-d_{i+2}(n+2) d_{i}(n+1) \\
& =\frac{2 i+4 n+9}{2(n+2)} d_{i+1}^{2}(n+1)+\frac{i+n+2}{n+2} d_{i}(n+1) d_{i+1}(n+1) \\
& \quad-\frac{2 i+4 n+11}{2(n+2)} d_{i}(n+1) d_{i+2}(n+1)-\frac{i+n+3}{n+2} d_{i}(n+1) d_{i+1}(n+1) \\
& =\frac{2 i+4 n+9}{2(n+2)} d_{i+1}^{2}(n+1)-\frac{2 i+4 n+11}{2(n+2)} d_{i}(n+1) d_{i+2}(n+1) \\
& >\frac{2 i+4 n+7}{2(n+2)} d_{i+1}^{2}(n+1)-\frac{2 i+4 n+11}{2(n+2)} d_{i}(n+1) d_{i+2}(n+1),
\end{aligned}
$$

which is positive by Lemma 2.2. It follows that for $0 \leq i \leq[(n+1) / 2]-1$,

$$
d_{i+1}(n+1) d_{i+1}(n+2)-d_{i+2}(n+2) d_{i}(n+1)>0 .
$$

This completes the proof of the first case.

The second case is when $[(n+1) / 2] \leq i \leq n-1$. From the recurrence relations (6) and (7), it follows that for $[(n+1) / 2] \leq i \leq n-1$, 


$$
\begin{aligned}
& d_{i+1}(n+2) d_{i+1}(n+1)-d_{i+2}(n+2) d_{i}(n+1) \\
& =\left(\frac{(4 n-2 i+5)(n+i+3)}{2(n+2)(n+1-i)} d_{i+1}(n+1)-\frac{(i+1)(i+2)}{(n+2)(n+1-i)} d_{i+2}(n+1)\right) \\
& \times\left(\frac{n+1+i}{n+1} d_{i}(n)+\frac{4 n+2 i+5}{2(n+1)} d_{i+1}(n)\right) \\
& -\left(\frac{n+3+i}{n+2} d_{i+1}(n+1)+\frac{4 n+2 i+11}{2(n+2)} d_{i+2}(n+1)\right) \\
& \quad \times\left(\frac{(4 n-2 i+3)(n+i+1)}{2(n+1)(n+1-i)} d_{i}(n)-\frac{i(i+1)}{(n+1)(n+1-i)} d_{i+1}(n)\right) \\
& =B_{1}(n, i) d_{i+1}(n+1) d_{i}(n)+B_{2}(n, i) d_{i+1}(n+1) d_{i+1}(n) \\
& \quad+B_{3}(n, i) d_{i+2}(n+1) d_{i}(n)+B_{4}(n, i) d_{i+2}(n+1) d_{i+1}(n),
\end{aligned}
$$

where $B_{1}(n, i), B_{2}(n, i), B_{3}(n, i)$ and $B_{4}(n, i)$ are given by

$$
\begin{aligned}
& B_{1}(n, i)=\frac{(n+i+3)(n+1+i)}{(n+2)(n+1-i)(n+1)}, \\
& B_{2}(n, i)=\frac{(n+i+3)\left(16 n^{2}+40 n+25+4 i\right)}{4(n+2)(n+1-i)(n+1)}, \\
& B_{3}(n, i)=-\frac{(n+1+i)\left(41+16 n^{2}+56 n-4 i\right)}{4(n+2)(n+1-i)(n+1)}, \\
& B_{4}(n, i)=-\frac{(i+1)(4 n+5-i)}{(n+2)(n+1-i)(n+1)} .
\end{aligned}
$$

Since $[(n+1) / 2] \leq i \leq n-1$, it follows from (3) that $d_{i+1}(n+1)>d_{i+2}(n+1)$ and $d_{i}(n)>d_{i+1}(n)$. Thus we get

$$
\begin{aligned}
d_{i+1}(n+1) d_{i}(n) & >d_{i+1}(n+1) d_{i+1}(n), \\
d_{i+1}(n+1) d_{i+1}(n) & >d_{i+2}(n+1) d_{i+1}(n) .
\end{aligned}
$$

Observe that $B_{1}(n, i)$ and $B_{2}(n, i)$ are positive, and $B_{3}(n, i)$ and $B_{4}(n, i)$ are negative. By the induction hypothesis (21) and inequalities (28) and (29), we find that, for $[(n+1) / 2] \leq i \leq n-1$,

$$
\begin{aligned}
d_{i+1}(n+2) & d_{i+1}(n+1)-d_{i+2}(n+2) d_{i}(n+1) \\
> & \left(B_{1}(n, i)+B_{2}(n, i)+B_{3}(n, i)+B_{4}(n, i)\right) d_{i+1}(n+1) d_{i+1}(n) \\
& =\frac{24 n+10 n^{2}-8 n i+8 i^{2}+13}{2(n+2)(n+1-i)(n+1)} d_{i+1}(n+1) d_{i+1}(n)>0 .
\end{aligned}
$$

From the inequalities (23) and (30), it follows that (22) holds for $0 \leq i \leq n-1$. 
It is still necessary to show that (22) is true for $i=n$, that is,

$$
\frac{d_{n}(n+1)}{d_{n+1}(n+1)}<\frac{d_{n+1}(n+2)}{d_{n+2}(n+2)} \text {. }
$$

For the recurrence relation (9), setting $i=n+2$, we find that

$$
\frac{d_{n}(n+1)}{d_{n+1}(n+1)}=\frac{2 n+3}{2}<\frac{2 n+5}{2}=\frac{d_{n+1}(n+2)}{d_{n+2}(n+2)},
$$

as desired. Hence the proof is complete by induction.

Lemmas 2.3 and 2.4 immediately imply the interlacing log-concavity of the Boros-Moll polynomials.

\section{Polynomials with triangular relations on coefficients}

Many combinatorial polynomials admit triangular relations on the coefficients. The log-concavity of polynomials of this kind has been extensively studied. We show that many classical polynomials of this kind are also interlacingly log-concave. For example, it is easy to check that the binomial coefficients, the Narayana numbers

$$
N(n, k)=\frac{1}{n}\left(\begin{array}{l}
n \\
k
\end{array}\right)\left(\begin{array}{c}
n \\
k+1
\end{array}\right),
$$

and the Bessel numbers

$$
B(n, k)=\frac{(2 n-k-1) !}{2^{k}(n-k) !(k-1) !}
$$

are interlacingly log-concave. Moreover, we give a criterion that applies to many combinatorial sequences such as the signless Stirling numbers of the first kind, the Stirling numbers of the second kind and the Whitney numbers.

Theorem 3.1. Suppose that for any $n \geq 0$,

$$
G_{n}(x)=\sum_{k=0}^{n} T(n, k) x^{k}
$$

is a polynomial of degree $n$ which has only real zeros, and suppose that the coefficients $T(n, k)$ satisfy a recurrence relation of the form

$$
T(n, k)=f(n, k) T(n-1, k)+g(n, k) T(n-1, k-1) .
$$

If

$$
\begin{aligned}
\frac{(n-k) k}{(n-k+1)(k+1)} f(n+1, k+1) & \leq f(n+1, k) \leq f(n+1, k+1), \\
g(n+1, k+1) \leq g(n+1, k) & \leq \frac{(n-k+1)(k+1)}{(n-k) k} g(n+1, k+1),
\end{aligned}
$$

then the polynomials $G_{n}(x)$ are interlacingly log-concave. 
Proof. Since the polynomial $G_{n}(x)$ has only real zeros, by Newton's inequality,

$$
k(n-k) T(n, k)^{2} \geq(k+1)(n-k+1) T(n, k-1) T(n, k+1) .
$$

Hence

$$
\begin{aligned}
& T(n, k) T(n+1, k+1)-T(n+1, k) T(n, k+1) \\
& =f(n+1, k+1) T(n, k) T(n, k+1)+g(n+1, k+1) T(n, k)^{2} \\
& \quad \quad-f(n+1, k) T(n, k) T(n, k+1)-g(n+1, k) T(n, k-1) T(n, k+1) \\
& \geq(f(n+1, k+1)-f(n+1, k)) T(n, k) T(n, k+1) \\
& \quad+\left(\frac{(n-k+1)(k+1)}{(n-k) k} g(n+1, k+1)-g(n+1, k)\right) T(n, k-1) T(n, k+1),
\end{aligned}
$$

which is positive by (32) and (33). It follows that

$$
\frac{T(n, k)}{T(n, k+1)} \geq \frac{T(n+1, k)}{T(n+1, k+1)} .
$$

On the other hand, we have

$$
\begin{aligned}
& T(n, k+1) T(n+1, k+1)-T(n, k) T(n+1, k+2) \\
& =f(n+1, k+1) T(n, k+1)^{2}+g(n+1, k+1) T(n, k) T(n, k+1) \\
& -f(n+1, k+2) T(n, k) T(n, k+2)-g(n+1, k+2) T(n, k+1) T(n, k) \\
& \geq\left(f(n+1, k+1)-\frac{(n-k-1)(k+1)}{(n-k)(k+2)} f(n+1, k+2)\right) T(n, k+1)^{2} \\
& \quad+(g(n+1, k+1)-g(n+1, k+2)) T(n, k+1) T(n, k) .
\end{aligned}
$$

It follows from (32) that

$$
\frac{T(n, k)}{T(n, k+1)} \leq \frac{T(n+1, k+1)}{T(n+1, k+2)}
$$

This completes the proof.

Employing Theorem 3.1, we can show that many combinatorial polynomials which have only real zeros are interlacingly log-concave, for example,

(1) the polynomials

$$
x(x+1)(x+2) \cdots(x+n-1),
$$

whose coefficients are the signless Stirling numbers of the first kind, which satisfy the recurrence relation

$$
c(n, k)=(n-1) c(n-1, k)+c(n-1, k-1) ;
$$


(2) the Bell polynomials whose coefficients are the Stirling numbers of the second kind $S(n, k)$, which satisfy the recurrence relation

$$
S(n, k)=S(n-1, k-1)+k S(n-1, k)
$$

(3) the Whitney polynomials

$$
W_{n}(x)=\sum_{k=0}^{n} W_{m}(n, k) x^{k},
$$

which have only real zeros; see [Benoumhani 1997; 1999]. The coefficients $W_{m}(n, k)$ satisfy the recurrence relation

$$
W_{m}(n, k)=(1+m k) W_{m}(n-1, k)+W_{m}(n-1, k-1) .
$$

\section{Acknowledgments}

We wish to thank the referee for valuable comments.

\section{References}

[Alvarez et al. 2001] J. Alvarez, M. Amadis, G. Boros, D. Karp, V. H. Moll, and L. Rosales, "An extension of a criterion for unimodality", Electron. J. Combin. 8:1 (2001), Research Paper 30. MR 2002g:05017 Zbl 0984.05008

[Amdeberhan and Moll 2009] T. Amdeberhan and V. H. Moll, "A formula for a quartic integral: a survey of old proofs and some new ones", Ramanujan J. 18:1 (2009), 91-102. MR 2009m:33008 Zbl 1178.33002

[Benoumhani 1997] M. Benoumhani, "On some numbers related to Whitney numbers of Dowling lattices”, Adv. in Appl. Math. 19:1 (1997), 106-116. MR 98f:05004 Zbl 0876.05001

[Benoumhani 1999] M. Benoumhani, "Log-concavity of Whitney numbers of Dowling lattices", Adv. in Appl. Math. 22:2 (1999), 186-189. MR 2000i:05008 Zbl 0918.05003

[Boros and Moll 1999a] G. Boros and V. H. Moll, "A criterion for unimodality", Electron. J. Combin. 6 (1999), Research Paper 10. MR 99k:05017 Zbl 0911.05004

[Boros and Moll 1999b] G. Boros and V. H. Moll, "An integral hidden in Gradshteyn and Ryzhik", J. Comput. Appl. Math. 106:2 (1999), 361-368. MR 2000c:33024 Zbl 0939.33007

[Boros and Moll 1999c] G. Boros and V. H. Moll, "A sequence of unimodal polynomials", J. Math. Anal. Appl. 237:1 (1999), 272-287. MR 2000m:33007 Zbl 0944.33009

[Boros and Moll 2001] G. Boros and V. H. Moll, "The double square root, Jacobi polynomials and Ramanujan's master theorem", J. Comput. Appl. Math. 130:1-2 (2001), 337-344. MR 2002d:33030 Zbl 1011.33005

[Boros and Moll 2004] G. Boros and V. H. Moll, Irresistible integrals: Symbolics, analysis and experiments in the evaluation of integrals, Cambridge University Press, 2004. MR 2005b:00001 Zbl 1090.11075

[Chen and Xia 2009] W. Y. C. Chen and E. X. W. Xia, "The ratio monotonicity of the Boros-Moll polynomials”, Math. Comp. 78:268 (2009), 2269-2282. MR 2010f:33046 Zbl 1221.33036 
[Chen et al. $\geq 2011]$ W. Y. C. Chen, S. X. M. Pang, and E. X. Y. Qu, "A combinatorial proof of the log-concavity of the Boros-Moll polynomials", to appear in Ramanujan J.

[Kauers and Paule 2007] M. Kauers and P. Paule, "A computer proof of Moll's log-concavity conjecture", Proc. Amer. Math. Soc. 135:12 (2007), 3847-3856. MR 2009d:33063 Zbl 1126.33009

[Moll 2002] V. H. Moll, "The evaluation of integrals: a personal story", Notices Amer. Math. Soc. 49:3 (2002), 311-317. MR 2002m:11105 Zbl 1126.11347

[Moll 2007] V. H. Moll, "Combinatorial sequences arising from a rational integral”, Online J. Anal. Comb. 2 (2007), Art. 4. MR 2008m:05006 Zbl 1123.05003

Received August 9, 2010. Revised May 12, 2011.

William Y. C. CHEN

CENTER FOR COMBINATORICS, LPMC-TJKLC

NANKAI UNIVERSITY

TIANJIN 300071

CHINA

chen@nankai.edu.cn

LARRY X. W. WANG

CENTER FOR COMBINATORICS, LPMC-TJKLC

NANKAI UNIVERSITY

TIANJIN 300071

CHINA

wxw@cfc.nankai.edu.cn

ERNEST X. W. XIA

CENTER FOR COMBINATORICS, LPMC-TJKLC

NANKAI UNIVERSITY

TIANJIN 300071

CHINA

xxwrml@mail.nankai.edu.cn 


\title{
PACIFIC JOURNAL OF MATHEMATICS
}

\author{
http://pacificmath.org \\ Founded in 1951 by \\ E. F. Beckenbach (1906-1982) and F. Wolf (1904-1989)
}

\section{EDITORS}

V. S. Varadarajan (Managing Editor)

Department of Mathematics

University of California

Los Angeles, CA 90095-1555

pacific@math.ucla.edu

Vyjayanthi Chari

Department of Mathematics

University of California

Riverside, CA 92521-0135

chari@math.ucr.edu

\section{Robert Finn}

Department of Mathematics Stanford University

Stanford, CA 94305-2125

finn@math.stanford.edu

Kefeng Liu

Department of Mathematics

University of California

Los Angeles, CA 90095-1555

liu@math.ucla.edu
Darren Long

Department of Mathematics

University of California

Santa Barbara, CA 93106-3080

long@math.ucsb.edu

Jiang-Hua Lu

Department of Mathematics

The University of Hong Kong

Pokfulam Rd., Hong Kong jhlu@maths.hku.hk

Alexander Merkurjev

Department of Mathematics

University of California

Los Angeles, CA 90095-1555

merkurev@math.ucla.edu
Sorin Popa

Department of Mathematics University of California

Los Angeles, CA 90095-1555 popa@math.ucla.edu

Jie Qing

Department of Mathematics

University of California

Santa Cruz, CA 95064

qing@cats.ucsc.edu

Jonathan Rogawski

Department of Mathematics

University of California

Los Angeles, CA 90095-1555

jonr@math.ucla.edu

\section{PRODUCTION}

pacific@math.berkeley.edu

\section{SUPPORTING INSTITUTIONS}

ACADEMIA SINICA, TAIPEI

CALIFORNIA INST. OF TECHNOLOGY INST. DE MATEMÁTICA PURA E APLICADA KEIO UNIVERSITY

MATH. SCIENCES RESEARCH INSTITUTE NEW MEXICO STATE UNIV.

OREGON STATE UNIV.

\author{
STANFORD UNIVERSITY \\ UNIV. OF BRITISH COLUMBIA \\ UNIV. OF CALIFORNIA, BERKELEY \\ UNIV. OF CALIFORNIA, DAVIS \\ UNIV. OF CALIFORNIA, LOS ANGELES \\ UNIV. OF CALIFORNIA, RIVERSIDE \\ UNIV. OF CALIFORNIA, SAN DIEGO \\ UNIV. OF CALIF., SANTA BARBARA
}

\author{
UNIV. OF CALIF., SANTA CRUZ \\ UNIV. OF MONTANA \\ UNIV. OF OREGON \\ UNIV. OF SOUTHERN CALIFORNIA \\ UNIV. OF UTAH \\ UNIV. OF WASHINGTON \\ WASHINGTON STATE UNIVERSITY
}

These supporting institutions contribute to the cost of publication of this Journal, but they are not owners or publishers and have no responsibility for its contents or policies.

See inside back cover or pacificmath.org for submission instructions.

The subscription price for 2011 is US \$420/year for the electronic version, and \$485/year for print and electronic.

Subscriptions, requests for back issues from the last three years and changes of subscribers address should be sent to Pacific Journal of Mathematics, P.O. Box 4163, Berkeley, CA 94704-0163, U.S.A. Prior back issues are obtainable from Periodicals Service Company, 11 Main Street, Germantown, NY 12526-5635. The Pacific Journal of Mathematics is indexed by Mathematical Reviews, Zentralblatt MATH, PASCAL CNRS Index, Referativnyi Zhurnal, Current Mathematical Publications and the Science Citation Index.

The Pacific Journal of Mathematics (ISSN 0030-8730) at the University of California, c/o Department of Mathematics, 969 Evans Hall, Berkeley, CA 94720-3840, is published monthly except July and August. Periodical rate postage paid at Berkeley, CA 94704, and additional mailing offices. POSTMASTER: send address changes to Pacific Journal of Mathematics, P.O. Box 4163, Berkeley, CA 94704-0163.

PJM peer review and production are managed by EditFLOW ${ }^{\mathrm{TM}}$ from Mathematical Sciences Publishers.

PUBLISHED BY PACIFIC JOURNAL OF MATHEMATICS

at the University of California, Berkeley 94720-3840

A NON-PROFIT CORPORATION

Typeset in LATEX

Copyright $(2011$ by Pacific Journal of Mathematics 


\section{PACIFIC JOURNAL OF MATHEMATICS}

Volume $254 \quad$ No. $1 \quad$ November 2011

A mean curvature estimate for cylindrically bounded submanifolds

Luis J. AlíAS and MARCos DAJCZER

Weyl group multiple Dirichlet series of type $C$

JENNIFER BEINEKE, BENJAMIN BRUBAKER and SHARON FrECHETTE

Milnor open books of links of some rational surface singularities

MOHAN BHUPAL and BURAK OZBAGCI

Simple closed curves, word length, and nilpotent quotients of free groups

KHALID BOU-RABEE and ASAF HADARI

Strong submodules of almost projective modules

GÁBOR BRAUN and JAN TRLIFAJ

Interlacing log-concavity of the Boros-Moll polynomials

William Y. C. Chen, LARry X. W. WAng and ERnest X. W. Xia

Schwarzian norms and two-point distortion

Martin Chuaqui, Peter Duren, William Ma, Diego Mejía, David

MINDA and BRAD OSGOOD

The principle of stationary phase for the Fourier transform of $D$-modules

JIANGXUE FANG

Monotonicity and uniqueness of a 3D transonic shock solution in a conic nozzle with

variable end pressure

JUN LI, ZHOUPING XIN and HUICHENG YIN

Refined open noncommutative Donaldson-Thomas invariants for small crepant resolutions

KENTARO NAGAO

The Dirichlet problem for harmonic functions on compact sets

TONY L. PERKINS

Extension of an analytic disc and domains in $\mathbb{C}^{2}$ with noncompact automorphism group

\section{MINJU SONG}

Regularity of the first eigenvalue of the $p$-Laplacian and Yamabe invariant along geometric flows

ER-MIN WANG and YU ZHENG 